\title{
Timoma do mediastino médio: relato de caso
}

\author{
Middle mediastinal thymoma: case report
}

Elias KALLÁS ${ }^{1}$, Alexandre C. HUEB² ${ }^{2}$ Ibrahim E. KALLÁS ${ }^{3}$, Alexandre C. KALLÁS ${ }^{3}$

RBCCV 44205-752

Resumo

Paciente do sexo feminino, 55 anos, branca, com sinais clínicos sugestivos de miastenia gravis há aproximadamente 3 meses. A prova terapêutica com neostigmina evidenciou melhora da disfagia e ptose palpebral. Na radiografia de tórax observou-se imagem ocupando o mediastino médio com projeção à direita. A tomografia computadorizada de tórax revelou a presença de massa no mediastino médio, sendo indicado o tratamento cirúrgico. Chamou nossa atenção a localização pouco usual, já que, preferencialmente, os timomas localizam-se no mediastino superior e anterior. A evolução pós-operatória foi boa, sem complicações.

Descritores: Timoma, cirurgia. Neoplasias do timo. Neoplasias do mediastino.
Trabalho realizado no Hospital das Clínicas Samuel Libânio Pouso Alegre - MG

1 - Professor Titular do Departamento de Cirurgia da Faculdade de Medicina da Universidade do Vale do Sapucaí UNIVÁS.

Pouso Alegre, MG

2 - Médico Assistente do Instituto do Coração do Hospital das clínicas da Faculdade de Medicina da Universidade de São Paulo - InCor.

São Paulo, SP

3 - Professor Assistente do Departamento de Cirurgia da Faculdade de Medicina da Universidade do Vale do Sapucaí - UNIVÁS

Pouso Alegre, MG

Endereço para correspondência:

Elias Kallás.

Rua Raquel de Paula Ribeiro, 7

37550-000 Pouso Alegre, MG

E-mail: eliaskallas@uol.com.br

\section{Abstract}

We report on a case of a 55-year-old female, patient, who presented with clinical signs suggestive of myasthenia gravis over a period of approximately 3 months. The therapeutic option using neostigmine gave an improvement of the dysphagia and palpebral ptosis. A chest radiograph image demonstrated a mass occupying the medium mediastinum with projection to the right. Computed tomography of the thorax revealed the presence of a mass in the medium mediastinum, and thus surgery was inicated. During the operation the was was observed in the medium mediastinum suggesting thymoma. What caught our attention was the unusual location of the tumor as normally thymomas are found in the upper and anterior mediastinum.

Descriptors: Thymoma, surgery. Thymus neoplasms. Mediastinal neoplasms.
Artigo recebido em fevereiro de 2005 Artigo aprovado em abril de 2005 


\section{INTRODUÇÃO}

O timoma é uma afecção rara, embora referido como o tumor mais freqüente do mediastino anterior, compreendendo aproximadamente 20 a 30\% das massas mediastinais em adultos [1]. Ocorre principalmente na $5^{\mathrm{a}}$ e $6^{\mathrm{a}}$ décadas da vida. São descritos dois tipos, o não invasivo, encapsulado (cerca de 2/3 dos casos) e o invasivo.

Em relação à sua localização, $75 \%$ ocupam o mediastino anterior, $15 \%$ o mediastino anterior e superior, $6 \%$ o mediastino superior e 4\% em outras localizações [2]. Recentemente foi publicado um caso de timoma do mediastino médio, que os autores rotularam como sendo o primeiro da literatura [3]. Posteriormente, outro caso foi relatado, em que o paciente foi tratado cirurgicamente com sucesso, sem necessidade de radioterapia adjuvante [4]. Revisão da literatura realizada por Minniti et al.[5] não evidenciou relatos de timomas em mediastino médio, estes autores descreveram um caso de timoma e um de cisto tímico nesta localização. Venuta et al. [6] enfatizaram o valor da timectomia no tratamento da miastenia gravis, sendo que $28,4 \%$ dos pacientes apresentavam timomas cuja presença influenciou negativamente o prognóstico do tratamento.

\section{RELATO DO CASO}

Paciente do sexo feminino, 55 anos, branca, com história de disfagia, ptose palpebral à esquerda e fraqueza muscular nos membros superiores há aproximadamente 3 meses. Ao exame físico, apresentava ausência de déficit motor ou alterações sensitivas, com força motora preservada Grau 3, Glasgow 15, reflexo cutâneo plantar em flexão bilateral, pupilas isocóricas e fotorreativas. Apresentava ainda dificuldade para cerrar a pálpebra esquerda. A prova terapêutica com neostigmina evidenciou melhora da disfagia e ptose palpebral. A paciente não fazia uso prévio de brometo de piridostigmina (mestinon). Os exames bioquímicos estavam normais. Na radiografia de tórax observou-se imagem ocupando o mediastino médio com projeção à direita. A tomografia computadorizada de tórax revelou a presença de massa no mediastino médio, sendo indicado o tratamento cirúrgico.

A paciente foi submetida à toracotomia mediana, visibilizando-se volumosa massa ocupando o mediastino médio em situação justa-pericárdica que iniciava-se logo abaixo da junção da veia cava superior com o átrio direito, estendendo-se lateralmente para a direita e, distalmente, em direção ao diafragma (Figura 1). Fato interessante foi a localização rara no mediastino médio.

Não havia aderência com estruturas mediastinais ou implantes pericárdicos e pleurais. A evolução pós-operatória foi satisfatória e a paciente recebeu alta no $5^{\circ}$ pós-operatório.
A tomografia computadorizada e a radiografia de tórax préoperatória demonstravam a localização do tumor (Figura 2). O exame anatomopatológico da peça, pela microscopia ótica e imuno-histoquímica, confirmou o diagnóstico de timoma.

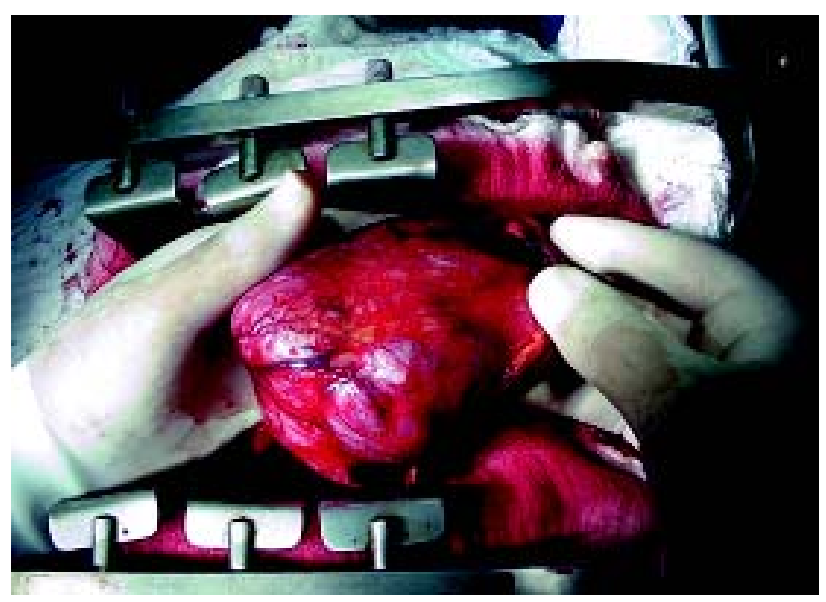

Fig. 1 - Timoma após descolamento ocupando o mediastino médio

(A)

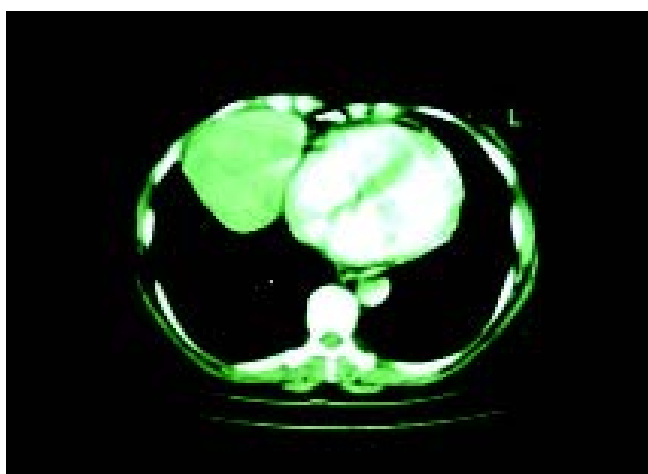

(B)

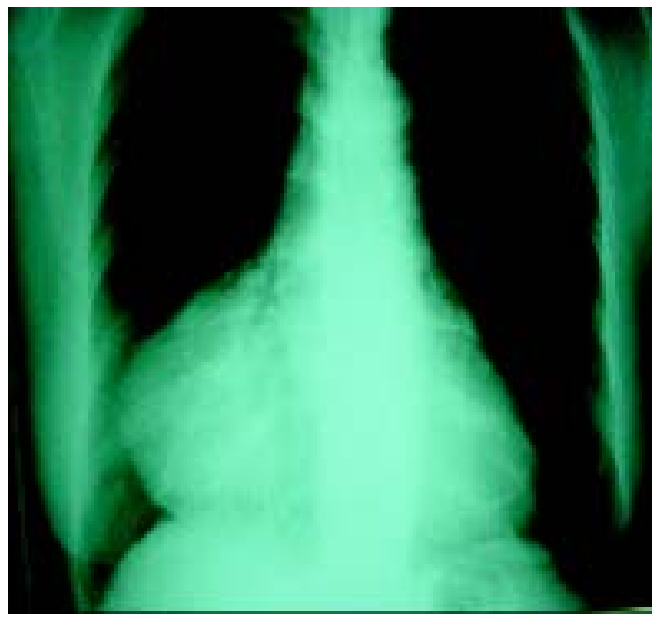

Fig. 2 - (A)Tomografia computadorizada e (B)radiografia de tórax pré-operatórias 


\section{COMENTÁRIOS}

Os timomas são tumores de crescimento lento e comportamse geralmente de forma benigna. Radiograficamente apresentam-se como estruturas redondas ou ovaladas, com margens bem definidas que podem conter alguma calcificação periférica. A associação com miastenia gravis ocorre em $50 \%$ dos pacientes com timoma. Por outro lado, apenas 15\% dos pacientes com miastenia gravis apresentam timoma. [7].

Uma vez identificado, o tratamento de escolha é a ressecção completa do timoma. Em nosso meio, o tumor benigno apresenta sobrevida em 10 anos de $82,6 \%$ e o maligno taxa média de sobrevida em 5 anos de 33,5\% [8].

$\mathrm{O}$ caso relatado chamou nossa atenção quanto à localização no mediastino médio, já que, preferencialmente, este tumor localiza-se no mediastino superior e anterior. Apesar da extensão por continuidade com tecidos remanescentes do timo localizados anterior e superiormente, a grande expansão tumoral, neste caso, se deu no mediastino médio, o que é uma raridade, sendo este um dos poucos casos relatados na literatura. O relato de Kojima et al. [3] descreve massa extrapleural localizada entre a veia cava superior e a traquéia, estendendo-se até a veia ázigos, abaixo da artéria inominada direita. Tal situação anatômica, ao nosso ver, não corresponde ao mediastino médio e sim ao mediastino superior [9]. Kanzaki et al. [4] relataram o caso de uma paciente de 60 anos, provavelmente o primeiro da literatura, localizado no mediastino médio. No mesmo ano, Minniti et al. [5] descreveram mais dois casos de massas neoplásicas no mediastino médio, sendo que apenas um tratava-se de timoma, o outro era um cisto tímico. A grande experiência de Venuta et al. [6] correlaciona os resultados da timectomia no tratamento da miastenia gravis, concluindo que os portadores de timoma apresentam prognóstico desfavorável. Ao que tudo indica, das 232 timectomias realizadas por estes autores, 62 timomas localizavam-se no mediastino superior e anterior, caso contrário teriam sido motivo de referência especial. O caso por nós apresentado identifica o timoma localizado no mediastino médio em posição justa-pericárdica, o que é extremamente raro. A paciente apresentava sinais e sintomas de miastenia gravis com resposta positiva à prova terapêutica. Através de esternotomia mediana foi retirado tumor de $11,50 \times 7,00 \times 4,00 \mathrm{~cm}$, ao lado do pericárdio, porém sem invadi-lo. No mesmo ato retirou-se também o tecido remanescente do timo, objetivando-se a cura da miastenia gravis. O exame anatomopatológico, pela microscopia ótica, foi sugestivo de timoma confirmado pelo estudo imunohistoquímico. A paciente foi encaminhada ao oncologista para tratamento coadjuvante quimio e radioterápico. Decorridos 28 meses após a intervenção, apresenta-se em boas condições e assintomática.

\section{REFERÊNCIAS BIBLIOGRÁFICAS}

1. Li JM, Zhou XM, Hu JG. Surgical treatment of 219 cases of primary tumor and cyst of the mediastinum. Hunan Yi ke Da Xue Xue Bao. 2001;26(2):149-51.

2. Ashour M. Prevalence of ectopic thymic tissue in myasthenia gravis and its clinical significance. J Thorac Cardiovasc Surg. 1995;109(4):632-5.

3. Kojima K, Yokoi K, Matsuguma H, Kondo T, Kamiyama Y, Mori K et al. Middle mediastinal thymoma. J Thorac Cardiovasc Surg. 2002;124(3):639-40.

4. Kanzaki M, Oyama K, Ikeda T, Yoshida T, Murasugi M, Onuki T. Noninvasive thymoma in the middle mediastinum. Ann Thorac Surg. 2004;77(6):2209-10.

5. Minniti S, Valentini M, Pinali L, Malago R, Lestani M, Procacci C. Thymic masses of the middle mediastinum: report of 2 cases and review of the literature. J Thorac Imaging. 2004;19(3):192-5.

6. Venuta F, Rendina EA, De Giacomo T, Della Rocca G, Antonini G, Ciccone AM et al. Thymectomy for myasthenia gravis: a 27-year experience. Eur J Cardiothorac Surg. 1999;15(5):6215.

7. Fraser RG, Pare JAP, Pare PD, Fraser RS, Genereux GP. Diagnosis of diseases of the chest. 3rd ed. Philadelphia:WB Saunders;1991. p.2820-31.

8. Pêgo-Fernandes PM, Ebaid GX, Galizia MS, Marchiori P, Suso FV, Jatene FB. Timoma: discussão sobre tratamento e prognóstico. J Pneumol 2001;27(6):288-94.

9. Di Dio LJA. Tratado de anatomia aplicada. 1a ed. São Paulo:Pólus Editorial;1999.p.308. 